\title{
Spectra optimizes the use of electron dose
}

Eric Van Cappellen ${ }^{1}$, Christian Maunders ${ }^{2}$, Ingrid Kieft ${ }^{2}$, Maarten Bischoff ${ }^{2}$, Felix Van Uden ${ }^{2}$, Mikhail Ovsyanko $^{3}$, Boy Markus ${ }^{3}$, Ruud Krijnen ${ }^{3}$, Cigdem Ozsoy-Keskinbora ${ }^{3}$, Bert Freitag ${ }^{3}$, Casper Smit $^{3}$, Veli Altin $^{3}$ and Rudolf Geurink ${ }^{3}$

${ }^{1}$ Thermo Fisher Scientific, 5350 NE Dawson Creek Drive, Hillsboro, OR 97124; USA, Portland, Oregon, United States, ${ }^{2}$ Thermo Fisher Scientific, Eindhoven, Noord-Brabant, Netherlands, ${ }^{3}$ Thermo Fisher Scientific, Achtseweg Noord 5, 5651 GG Eindhoven, The Netherlands, Eindhoven, Noord-Brabant, Netherlands

The analytical scanning transmission electron microscope is being used today to investigate a wider than ever variety of material types and to extract, in the most efficient way possible, more information than ever before. This places demands on the microscope to accommodate a wide range of detectable beam currents, convergence and collection angles, detector and aperture geometries and spectroscopies whilst maintaining high contrast, interpretable and sub- $\AA$ imaging conditions. More than ever the accelerating voltage is playing a crucial role in obtaining the best characterization of increasingly complex materials. This role varies between enabling high spatial resolution imaging, mitigation of electron radiation damage effects [1], as well as optimizing the detectability limits in spectroscopic studies [2].

Technological advances have been engineered into the new Spectra system enabling effortlessly changing the accelerating voltage for optimized imaging and then analysis during a single microscopy experimental session. These advances in functionality are coupled with corresponding advances in electron and X-ray detectors all of which are aimed at making better use of the electrons that have interacted with the specimen.

Figure 1 succinctly illustrates the mitigation of electron sputtering damage by optimization of the experimental conditions in the Spectra system. Figure 1a) is an ultra high resolution image, which is achieved using an electron beam energy of $300 \mathrm{keV}$, however, as is dictated by fundamental physics, this is accompanied by a small amount of sputtering damage as discussed in detail by Egerton. Because of the efficient and new advanced technology integrated into Spectra, the same area is imaged under identical beam current conditions, although at a reduced beam energy of $200 \mathrm{keV}$ in Figure 1b). This operational mode is accessible for high resolution spectroscopy within 5 minutes of changing the experimental conditions. Under these optimized spectroscopy conditions, no discernible sputtering is recorded, with only a minor reduction in resolution. Importantly, the benefits of Spectra's enhanced x-ray spectrometers [3] are allowed to bring their full power to bare on the characterization of the material. 
$300 \mathrm{kV}$

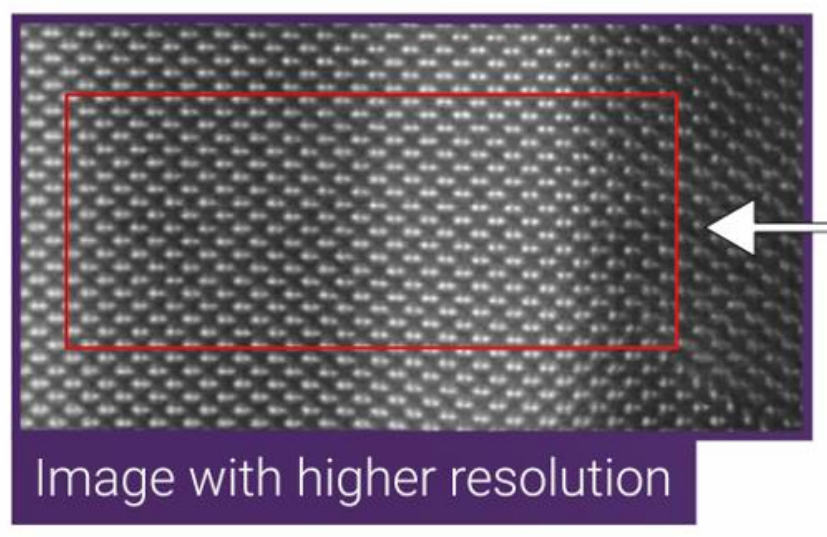

$200 \mathrm{kV}$

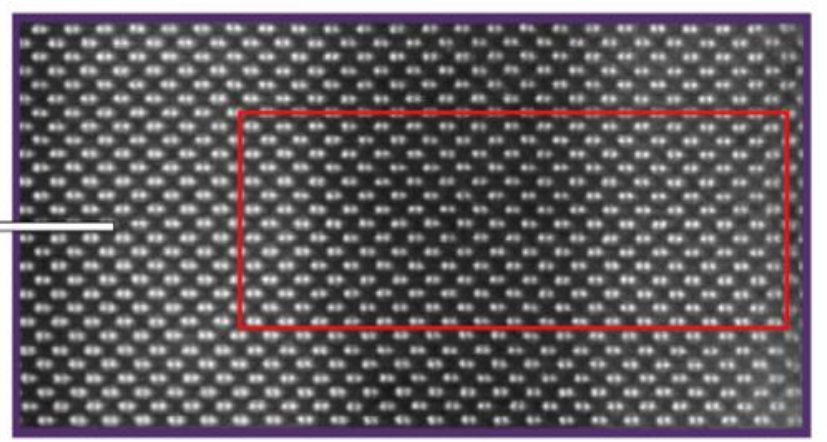

-
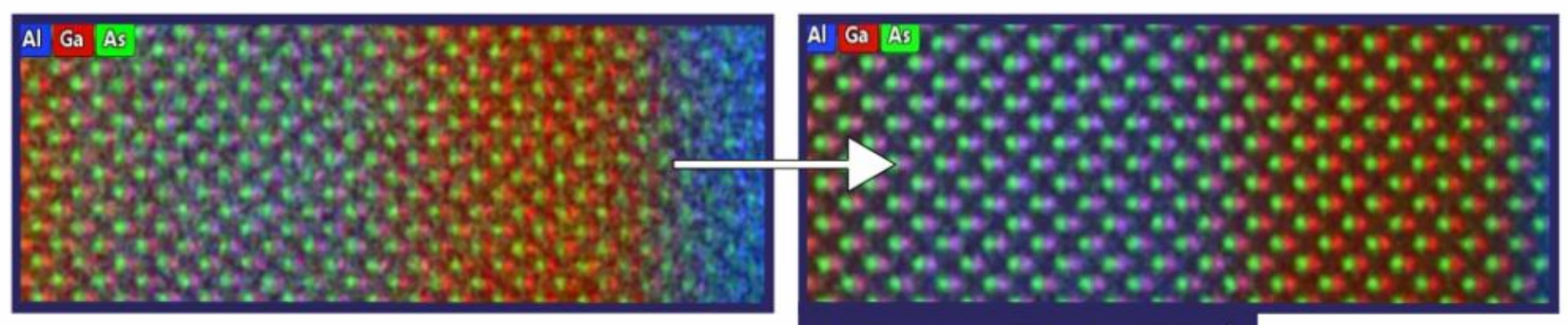

Improve EDX signal
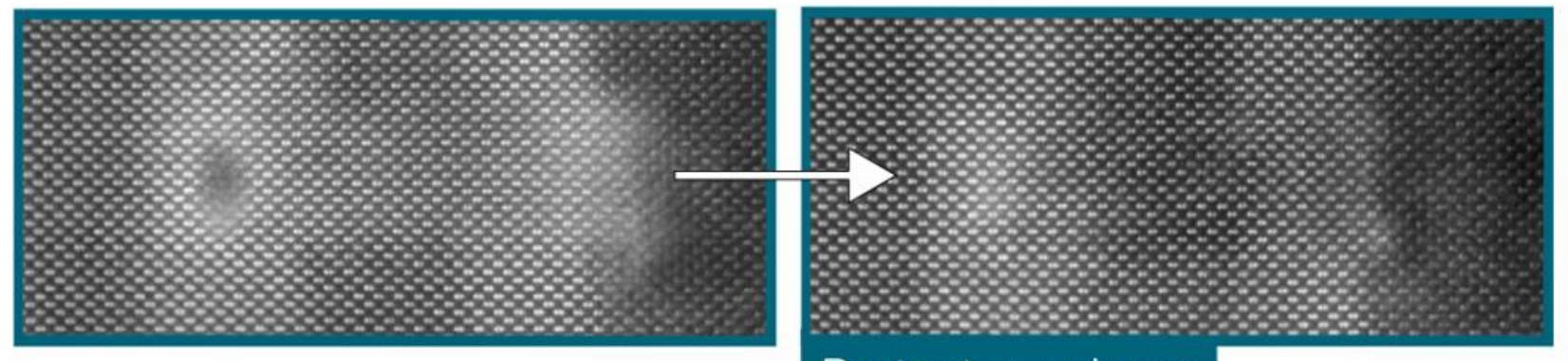

Protect specimen

Figure 1. Al-Ga-As multilayer sample courtesy of J. Zweck, University of Regensburg; data collected by Cigdem Ozsoy-Keskinbora. The combined 300kV and 200kV imaging and EDX mapping experiment was performed in less than 1 hour.

\section{References}

[1] Egerton R.F. et al., Micron (2004) V35, 399

[2] Zaluzec, N.J. Ultramicroscopy (2019) V203, 163

[3] Zaluzec, N.J. Submitted Proceedings of Microscopy \& Microanalysis 2021. 